\title{
Fisioterapia na melhora da depressão e ansiedade: revisão de literatura
}

\author{
Physiotherapy in improving depression and anxiety: literature review \\ Fisioterapia en la mejora de la depresión y ansiedad: revisión de la literatura
}

Recebido: 12/11/2021 | Revisado: 16/11/2021 | Aceito: 21/11/2021 | Publicado: 29/11/2021

\author{
Carla Noimann \\ ORCID: https//orcid.org/0000-0002-3020-4843 \\ Centro Universitário da Amazônia, Brasil \\ E-mail:noimanncarla@gmail.com \\ Filipe de Sousa Morais \\ ORCID: https//orcid.org/0000-0001-7257-903X \\ Centro Universitário da Amazônia, Brasil \\ E-mail: Felipe.morais235@icloud.com \\ Natasha Souza Silva dos Santos \\ ORCID: https//orcid.org/0000-0003-1806-1496 \\ Centro Universitário da Amazônia, Brasil \\ E-mail: nashata01@gmail.com \\ Naira Patricia Castro de Oliveira \\ ORCID: https//orcid.org/0000-0003-1896-7693 \\ Centro Universitário da Amazônia, Brasil \\ E-mail:nairacastro@outlook.com
}

\begin{abstract}
Resumo
Introdução: Segundo a OMS (Organização mundial de saúde) estima-se que no Brasil tem 12 milhões de pessoas (em torno de 5,8 \% da população) sofrem de depressão e consequentemente adquirem a ansiedade. Esse quadro se agrava pela falta de conhecimento sobre o assunto e pela falta recursos para os devidos tratamentos, infelizmente os métodos de tratamento ainda são extremamente invasivos através de fortes medicamentos. Metodologia: Esse estudo foi baseado em uma pesquisa bibliográfica, de caráter exploratório, por meio de uma pesquisa literária, a partir da análise de artigos e livros a respeito do tema, nas seguintes bases de dados: PunMed, Google acadêmico, scielo e Pedro, selecionados nos últimos 20 anos, com os temas ansiedade, depressão, massagem e acupuntura. Resultados: A partir desse estudo bibliográfico comprovamos a eficácia da fisioterapia no tratamento de ansiedade e depressão, utilizando de meios alternativos como: a acupuntura e a massoterapia. Na aplicação de tais técnicas foi relatado a diminuição dos sintomas dessas patologias. É de suma importância que o paciente entenda que o medicamento não é única forma de tratamento. Considerações finais: Podemos concluir que apesar de serem pouco citados, tratamentos alternativos para depressão e ansiedade são eficazes, desde que sejam aplicados e receitados por profissionais qualificados.
\end{abstract}

Palavras-chave: Ansiedade; Acupuntura; Depressão; Massagem.

\begin{abstract}
Introduction: According to the WHO (World Health Organization) it is estimated that in Brazil 12 million people (around 5.8\% of the population) suffer from depression and consequently acquire anxiety. clinical presentation is aggravated by the lack of knowledge on the theme and by the lack of resources for the proper treatments, unfortunately the treatment methods are still extremely invasive through strong medications. Methodology: This study was based on an exploratory bibliographical research, through a literary research, from the analysis of articles and books on the theme, in the following databases: PunMed, Google academic, Scielo and Pedro, selected in the last 20 years, with the themes of anxiety, depression, massage and acupuncture. Results: Based on this bibliographical study, we prove the effectiveness of physical therapy in the treatment of anxiety and depression, using alternative means such as acupuncture and massage therapy. In the application of such techniques, a decrease in the symptoms of these pathologies has been reported. It is extremely important that the patient understands that medication is not the only form of treatment. Final considerations: We can conclude that despite being little mentioned, alternative treatments for depression and anxiety are effective, as long as they are applied and prescribed by qualified professionals.
\end{abstract}

Keywords: Anxiety; Acupuncture; Depression; Massage.

\section{Resumen}

Introducción: Según la OMS (Organización Mundial de la Salud) se estima que 12 millones de personas en Brasil (alrededor del 5,8\% de la población) padecen depresión y, en consecuencia, adquieren ansiedad. Este cuadro se ve agravado por la falta de conocimiento sobre el tema y por la falta de recursos para los tratamientos adecuados, lamentablemente los métodos de tratamiento continúan siendo extremadamente invasivos a través de medicamentos fuertes. Metodología: Este estudio se basó en una investigación bibliográfica exploratoria, a través de una investigación 
literaria, a partir del análisis de artículos y libros sobre el tema, en las siguientes bases de datos: PunMed, Academic Google, scielo y Pedro, seleccionados en los últimos 20 años, con los temas de ansiedad, depresión, masajes y acupuntura. Resultados: A partir de este estudio bibliográfico, se verificó la efectividad de la fisioterapia en el tratamiento de la ansiedad y la depresión, utilizando medios alternativos como la acupuntura y la terapia de masajes. En la aplicación de tales técnicas se ha reportado una disminución de los síntomas de estas patologías. Es extremadamente importante que el paciente comprenda que la medicación no es la única forma de tratamiento. Consideraciones finales: Podemos concluir que, a pesar de ser poco mencionados, los tratamientos alternativos para la depresión y la ansiedad son efectivos, siempre y cuando sean aplicados y prescritos por profesionales calificados.

Palabras clave: Ansiedad; Acupuntura; Depresión; Masaje.

\section{Introdução}

A depressão e a ansiedade são umas das principais doenças que acometem a sociedade. Segundo a OMS (Organização mundial de saúde) estima-se que no Brasil tem 12 milhões de pessoas (em torno de 5,8 \% da população) sofrem de depressão e consequentemente adquirem a ansiedade. Sendo a maior taxa da América Latina e a segunda maior das Américas, atrás apenas dos Estados Unidos na mortalidade (Stopa et al, 2015).

A depressão caracteriza-se como um transtorno mental, um quadro clínico que apresenta como principalmente característica um grande sentimento de tristeza e a perda de interesse pelas coisas do dia-a-dia. O paciente sente como se nada fizesse algum sentindo em sua vida. Esses são exemplos de complicações que se agravam pela falta de conhecimento sobre o assunto e pela falta recursos para os devidos tratamentos. (OMS, 2008)

A prevalência da depressão gera em torno dos países de baixa renda, e com o aumento da super população a qualidade de vida vem diminuindo diariamente trazendo consigo diversos problemas de saúde. Consequentemente o transtorno também está associado ao aumento de risco para doenças cardíacas coronarianas, abuso de álcool e outas substâncias que causam danos. (Minayo, et al, 2021)

Outra patologia que também está relacionada ao fator psicológico é ansiedade que envolve considerações como diferenciar ansiedade normal da ansiedade patológico, e um meio prático de avaliar são através da reação ansiosa se é de curta duração ou prolongada. O Brasil lidera, com 7,3\% da população com transtornos de ansiedade, isso se dá devido a atitudes obsessivas e compulsivas, estresse pós-traumático, ataques de pânico e fobias. (Costa et al, 2019)

Assim como a depressão, a ansiedade é um transtorno em que esses sintomas são primários, não deriva de outras patologias através do medo é quando um indivíduo fica em um estado emocional de nervosismo e preocupações desconfortáveis que chega a ser perturbador; suas causas são devidas não ter controle sobre suas emoções. (Castillo, et al, 2000)

A ansiedade não está ligada com o fato em si da ameaça; ela pode ocorrer por antecipação, antes da ameaça, persistir depois que a ameaça encerrou, ou ocorrer sem ameaça identificáveis. A ansiedade é muitas vezes é acompanhada por alterações físicas e comportamentais similares ao um ataque de pânico. (Barnhill, 2018)

O profissional fisioterapeuta tem autonomia e a competência para executar diversas atividades estabelecer os parâmetros através de uma avaliação, diagnósticos fisioterapêuticos, atuar também no planejamento e programações de ações preventivas, além de educação em saúde também faz a intervenção no dia a dia, através de gerenciamento de serviços de saúde. (Ribeiro, 2014)

O tratamento para depressão e ansiedade se dá principalmente através de medicamentos que na sua maioria das vezes são invasivos e prejudiciais à saúde causando dependência, isso acaba fazendo com que os pacientes procurem meio alternativos para o tratamento, tais como exemplo: massoterapia e acupuntura. (Razzouk, 2016)

A massagem tem uma longa história e com o passar dos anos suas técnicas foram aprimoradas, mas não perderam sua essência, que seria: o tratamento através do toque das mãos. Com isso vale ressaltar a importância do toque para eficácia do 
tratamento e com a massagem não foi diferente porque através dela o paciente entra em um estado de relaxamento e assim aliviando suas dores, promovendo muitas das vezes uma diminuição de estresse emocionais. (Abreu, Souza, \& Fangundes, 2012).

A acupuntura é uma técnica chinesa que chegou ao Brasil através da imigração japonesa, é um procedimento que nos tempos atuais foi ganhando destaque por ser um tratamento inovador e alternativo. Suas características se dão principalmente pelos seus efeitos analgésicas e anti-inflamatória fazendo com que o paciente se sinta mais disposto fisicamente e espiritualmente. (Santos, 2010).

A acupuntura tem demonstrado ser eficiente no tratamento de doenças psiquiátricas como a ansiedade e depressão, ganhando espaço e aceitação no meio científico pela utilização da técnica como meio de tratamento e tendo o objetivo de diminuir o consumo de antidepressivos. A Acupuntura é uma das formas mais antigas de terapia na antiga filosofia chilena, ao longo de vários anos a medicina chilena mostra que todas as desordens são descritas em pontos específicos na superfície da pele ou exatamente abaixo (Trindade, 2017).

Com a inclusão manual de agulhas, sendo utilizando vários adjuntos com frequência assim como a acupuntura elétrica (Eletroacupuntura), utilizando um estimular elétrico conectado a agulha de acupuntura. A Acupuntura com moxibustão ou moxaterapia com o objetivo de aplicar calor direta ou indiretamente sobre a pele através de um bastão enrolado com ervas medicinais (Fernando, 2013).

\section{Justificativa}

Embora o tema ansiedade e depressão sejam abordados por diversos meios de pesquisa, ainda existem muitos questionamentos acerca dos benefícios procedentes aos exercícios de tratamento em relação aos distúrbios. Através de um estudo aprofundado temos a fisioterapia como uma das formas de tratamento, que buscam meios do dia-a-dia para facilitar o desenvolvimento do tratamento em relação ansiedade e depressão.

Trazer métodos alternativos para tratar a depressão e ansiedade onde atualmente os meios mais utilizados e considerados eficazes eram invasivos (remédios controlados com efeitos colaterais), tem-se a necessidade de conhecimento aprofundado onde preveja um tratamento de forma eficiente e seja menos agressivo. E através desses questionamentos buscamos um tema onde não era muito avaliado a perspectiva fisioterapêutica diante do problema.

\section{Metodologia}

Para Cordeiro et al (2007) "a revisão da literatura narrativa ou tradicional, quando comparada à revisão sistemática, apresenta uma temática mais aberta; dificilmente parte de uma questão específica bem definida, não exigindo um protocolo rígido para sua confecção; a busca das fontes não é pré-determinada e específica, sendo frequentemente menos abrangente."

A seleção dos estudos tem como o objetivo trazer uma revisão atualizada do conhecimento sobre um assunto especifico e a interpretação das informações podem estar sujeitas à subjetividade dos autores. É fundamental para a teoria de artigos, dissertações, teses, trabalhos de conclusão de cursos. (Unesp, 2015)

Neste estudo, utilizou-se o método de pesquisa bibliográfica, a partir de análise de artigos e documentos a respeito do tema, nas seguintes bases de dados: PunMed, Google acadêmico, scielo e Pedro, selecionados nos últimos 20 anos, com os temas ansiedade, depressão, massagem e acupuntura.

Foram incluídos nessa revisão pesquisas que abordavam métodos de tratamento contra a ansiedade e depressão, tais como a acupuntura e massoterapia. O estudo aborda de modo geral várias perspectivas em relação a essas duas patologias, fazendo com que ocorra uma leitura de fácil entendimento. 
A pesquisa bibliográfica de uma forma narrativa é um dos principais meios de adquirir conhecimentos entre os acadêmicos, sendo eles obrigatórios em trabalhos científicos. Através dela e feito uma coleta dados por meios de artigos, revistas cientificas e livros para utilizar como embasamento na pesquisa.

Para Lima e Mioto (2007), "É possível afirmar que para a realização de uma pesquisa bibliográfica é imprescindível seguir por caminhos não aleatórios, uma vez que esse tipo de pesquisa requer alto grau de vigilância epistemológica, de observação e de cuidado na escolha e no encaminhamento dos procedimentos metodológicos.

\section{Resultados}

Os resultados que tivemos por meios dessa revisão bibliográfica comprova a eficácia da fisioterapia na amenização dos sintomas da ansiedade e depressão,por meios alternativos como acupuntura e massagem. Esses estudos mostram a importância de uma alternativa menos invasiva para tratamentos psicólogos.

O resultado dessa revisão foi constituído por cinco artigos selecionadosnas plataformas Publisher, Scielo e Google acadêmico. Ouve uma grande dificuldade durante as pesquisas por ser um assunto pouco falado, de vários artigos encontrados apenas vinte entravam nos nossos critériose assim selecionamos apenas cinco que são apresentados no Quadro 1, a seguir:

Quadro 1. Artigos selecionados.

\begin{tabular}{|c|c|c|c|c|c|}
\hline Autor & Fonte & Titulo & Objetivo & Metodologia & Conclusão \\
\hline $\begin{array}{l}\text { Takeda et al. } \\
2006\end{array}$ & Publisher & $\begin{array}{l}\text { Atividade } \\
\text { física, saúde } \\
\text { mental e } \\
\text { reabilitação } \\
\text { psicossocial. }\end{array}$ & $\begin{array}{l}\text { Nosso objetivo foi } \\
\text { verificar a produção } \\
\text { científica disponível } \\
\text { em relação à atividade } \\
\text { física, saúde mental e } \\
\text { reabilitação } \\
\text { psicossocial de forma } \\
\text { integrada } \\
\text { relacionada. }\end{array}$ & $\begin{array}{l}\text { Estudou-se a produção } \\
\text { científica disponível integrando } \\
\text { ou relacionando os temas } \\
\text { atividade física, saúde mental e } \\
\text { reabilitação psicossocial. } \\
\text { Foram pesquisadas as bases de } \\
\text { dados: MEDLINE, LILACS e } \\
\text { SciELO, utilizando-se os } \\
\text { descritores: "reabilitação } \\
\text { psicossocial", "saúde mental" e } \\
\text { "atividade física" }\end{array}$ & $\begin{array}{l}\text { Embora haja escassez de publicações integrando } \\
\text { os temas em foco, percebeu-se um aumento } \\
\text { gradativo dessas. Os estudos existentes } \\
\text { evidenciam a contribuição da atividade física } \\
\text { programada para manutenção e recuperação da } \\
\text { saúde em geral e da saúde mental. Torna-se } \\
\text { necessário, portanto, a integração do profissional } \\
\text { de educação física à equipe de saúde mental e a } \\
\text { realização de estudos sobre essa temática, } \\
\text { visando concretizar a assistência integral baseada } \\
\text { em evidências. }\end{array}$ \\
\hline $\begin{array}{l}\text { Goyatá et al. } \\
2016\end{array}$ & Scielo & $\begin{array}{ll}\text { Efeitos } & \text { da } \\
\text { acupuntura } & \text { no } \\
\text { tratamento } & \text { da } \\
\text { ansiedade: } & \\
\text { revisão } & \\
\text { integrativa } & \end{array}$ & $\begin{array}{l}\text { Avaliar as evidencias } \\
\text { cientificas disponíveis } \\
\text { na literatura sobre os } \\
\text { efeitos da acupuntura } \\
\text { no tratamento da } \\
\text { ansiedade e a } \\
\text { qualidade desses } \\
\text { estudos }\end{array}$ & $\begin{array}{l}\text { Revisão integrativa, realizada } \\
\text { nas bases/bancos de dados } \\
\text { CINAHL, } \\
\text { PUBMEDPICO, SciELO, The } \\
\text { Cochrane Library, no período } \\
\text { entre 2001 a 2014. Os } \\
\text { descritores anxiety, } \\
\text { acupuncture therapy, } \\
\text { acupuncture e anxiety disorders } \\
\text { foram combinados entre si para } \\
\text { garantir a ampla busca de } \\
\text { estudos primários e anxiety } \\
\text { disorders foram combinados } \\
\text { entre si para garantir a ampla } \\
\text { busca de estudos primários. }\end{array}$ & $\begin{array}{l}\text { A acupuntura parece ser um tratamento } \\
\text { promissor para a ansiedade, no entanto, há } \\
\text { necessidade de melhorar a qualidade } \\
\text { metodológica das pesquisas nessa temática. }\end{array}$ \\
\hline $\begin{array}{l}\text { Carvalho et } \\
\text { al. } \\
2018\end{array}$ & $\begin{array}{l}\text { Google } \\
\text { acadêmico }\end{array}$ & $\begin{array}{l}\text { Efeitos da } \\
\text { massoterapia } \\
\text { sobre o sistema } \\
\text { imunológico }\end{array}$ & $\begin{array}{l}\text { Objetivo principal } \\
\text { sistematizar com base } \\
\text { na literatura os efeitos } \\
\text { da massoterapia no } \\
\text { sistema imunológico }\end{array}$ & $\begin{array}{l}\text { Utilizou-se de artigos e } \\
\text { bibliografias do ano de } 2001 \text { até } \\
2015 \text {. Onde foi realizada uma } \\
\text { revisão sistemática da } \\
\text { literatura, e seus artigos foram } \\
\text { coletados da base de banco de } \\
\text { dados: Google Acadêmico e } \\
\text { SciELO, a partir dos } \\
\text { descritores: massoterapia, } \\
\text { sistema imunológico. A } \\
\text { amostra foi empregada de dez } \\
\text { trabalhos. }\end{array}$ & $\begin{array}{l}\text { As publicações analisadas descreveram as } \\
\text { principais técnicas de massoterapia, bem como } \\
\text { seus efeitos no organismo humano, destacando } \\
\text { sua ação antinflamatória e consequentemente no } \\
\text { sistema imunitário. }\end{array}$ \\
\hline
\end{tabular}




\begin{tabular}{|c|c|c|c|c|c|}
\hline $\begin{array}{l}\text { Picolli da } \\
\text { Silva } \\
2008\end{array}$ & Scielo & $\begin{array}{l}\text { O tratamento } \\
\text { da ansiedade } \\
\text { por intermédio } \\
\text { da acupuntura: } \\
\text { um estudo de } \\
\text { caso }\end{array}$ & $\begin{array}{l}\text { O objeto deste artigo é } \\
\text { relatar o tratamento } \\
\text { realizado por meio da } \\
\text { acupuntura a uma } \\
\text { paciente que } \\
\text { apresentava transtorno } \\
\text { de ansiedade. }\end{array}$ & $\begin{array}{l}\text { Após a identificação dos } \\
\text { sintomas de ansiedade, } \\
\text { realizados pela queixa da } \\
\text { paciente e pela análise clínica } \\
\text { embasada no DSM IV, foram } \\
\text { realizadas } 10 \text { sessões de } \\
\text { acupuntura tradicional chinesa, } \\
\text { utilizando como referencial } \\
\text { teórico a literatura clássica da } \\
\text { medicina chinesa. }\end{array}$ & $\begin{array}{l}\text { Os resultados obtidos foram a diminuição parcial } \\
\text { dos sintomas a partir da quarta sessão e uma } \\
\text { significativa melhora da paciente, com o relato } \\
\text { do alívio dos sintomas a partir da sexta sessão de } \\
\text { tratamento. }\end{array}$ \\
\hline $\begin{array}{l}\text { Seubert et al } \\
2008\end{array}$ & $\begin{array}{l}\text { Google } \\
\text { acadêmico }\end{array}$ & $\begin{array}{l}\text { A massagem } \\
\text { terapêutica } \\
\text { auxiliando na } \\
\text { prevenção e } \\
\text { tratamento das } \\
\text { doenças físicas } \\
\text { e psicológicas }\end{array}$ & $\begin{array}{l}\text { O artigo tem como } \\
\text { objetivo evidenciar os } \\
\text { benefícios na } \\
\text { massoterapia } \\
\text { prevenção na } \\
\text { tratamento de doenças, } \\
\text { promovendo alívio de } \\
\text { dores e doenças } \\
\text { psicossomáticas } \\
\text { segundo a visão } \\
\text { reichiana, permitindo } \\
\text { a flexibilização das } \\
\text { couraças ou tensões } \\
\text { crônicas no corpo. }\end{array}$ & $\begin{array}{l}\text { São destacados estudos sobre a } \\
\text { melhora da imunidade, } \\
\text { aumento de endorfinas, alívio } \\
\text { de sintomas como enxaqueca, } \\
\text { depressão, } \\
\text { bronquite, entre ansiedade, } \\
\text { Enfatiza-se também atros. } \\
\text { importância da associação de } \\
\text { terapias -masso e psicoterapia } \\
\text { para facilitar } \\
\text { desenvolvimento da cura nos } \\
\text { níveis físico e psicológico. }\end{array}$ & $\begin{array}{l}\text { A massoterapia pode também acrescentar em } \\
\text { uma melhor qualidade de vida e auxílio às } \\
\text { terapias corporais, se ambas trabalharem juntas, } \\
\text { (psicoterapeuta e massoterapeuta). Estas } \\
\text { terapias, que são complementares visam mostrar } \\
\text { ao sujeito que ele pode voltar a ter prazer na vida } \\
\text { e ser alguém mais saudável em nível físico e } \\
\text { mental e mais consciente na sua vida. }\end{array}$ \\
\hline
\end{tabular}

Fonte: Autores.

\section{Discussão}

Para Carvalho et al. (2018), a massagem promove através da manipulação dos edemas corporais, a evacuação das toxinas através da urina e das fezes, atua no aumento de sangue circulante local, liberando as células gordurosas. Ela é muito eficaz no tratamento do estresse, pois uma vez que ela atua nos tecidos consegue oxigená-los de forma eficiente, devolvendo seus nutrientes e reparando o cansaço. Este processo vai se dando aos poucos na medida em que as veias e a linfa vão trabalhando normalmente. Além disso, quando há a liberação das toxinas, consequentemente se vê a melhora na circulação sanguínea que promove melhora do quadro de ansiedade e também atua nos fatores do rejuvenescimento. E que há alguns fatores que influenciam diretamente na massagem são eles: a direção do movimento, a intensidade da pressão, a frequência e o ritmo dos movimentos, os meios a ser utilizada, a posição correta, como também a duração e a frequência do tratamento.

De acordo com Goyatá et al. (2016), os efeitos da acupuntura no tratamento da ansiedade têm se mostrado significativos se comparados aos tratamentos convencionais; no entanto, os estudos de ensaios clínicos randomizados apresentam baixa qualidade metodológica. O Quality Score for Acupuncture Trials é um instrumento que pode auxiliar na avaliação da qualidade desses estudos e na elaboração de conclusões clínicas relevantes em futuras revisões. Isso porque o nível ou força de evidência por si só não garante a qualidade das pesquisas. Evidências científicas mostram que essa terapia é promissora e sua incorporação no tratamento da ansiedade na prática clínica de enfermagem, no contexto do Sistema Único de Saúde, poderá contribuir para a redução do tratamento farmacológico e o seu uso indiscriminado e prolongado, evitando aos pacientes prejuízos ou mesmo a morte.

Já Takeda et al. (2006), destaca que a atividade física realizada com regularidade e moderada intensidade contribui para a manutenção e recuperação da saúde em geral e está positivamente associada à saúde mental e social, com mudanças saudáveis no estilo de vida, consequentemente melhorando sua qualidade. A atuação da atividade física nos domínios motor, afetivo-social e cognitivo, contribui com uma parcela importante no processo de reabilitação psicossocial. Além de reduzir, pode também prevenir declínios funcionais associados à doença mental, e ainda, ajudar no aumento da autoestima, na criatividade, na socialização e possibilitando a melhora da qualidade de vida. Porém a necessidade de mais investigações controladas, pois apesar das evidências positivas, elas não são suficientes para a completa descrição do mecanismo neurobiológico envolvido nas mudanças havidas. 
Segundo Picoli (2008), a ansiedade é um fenômeno amplamente estudado no ocidente pela Psicologia, pela psicanálise e pela Medicina. caracteriza-se por um estado subjetivo desagradável de inquietação, tensão e apreensão. Embora a terminologia ansiedade não seja encontrada na literatura da medicina tradicional chinesa, os sintomas descritos são amplamente estudados. Tal literatura considera essa patologia metaforicamente uma desarmonia do espírito, e apresenta uma série de possibilidades de intervenções por técnicas tradicionais como a acupuntura.

Para Seubert et al (2008) A terapia através da massagem pode auxiliar o trabalho do psicoterapeuta, na eliminação ou amenização de diversos estados mentais, como: a depressão, ansiedade, dentre outras. De forma geral, os benefícios gerais da massagem ocidental e oriental são: melhora a percepção e sensibilidade de seu corpo, amplia a consciência corporal e o cuidado ofertado durante a sessão de massagem gera maior confiança e sensação de acolhimento e bem-estar. Outros benefícios como diminuição da ansiedade, da carência emocional, amenização no uso de drogas como álcool, tabaco.

\section{Considerações Finais}

A partir desta revisão bibliografiva podemos concluir que é pouco falado sobretratamentos alternativos para doenças psicologicas como depressão e ansiedade e os recursos ainda são limitados. A principal opção de tratamento ainda é utilização de medicamentos que geralmente são controlados, ou seja, trazem efeitos colaterais.

Embora existam vários tratamentos farmaceuticos bem estabelecidos para a ansiedade e a depressão, muitos pacientes obtem uma boa resposta ou acabam sofrendo efeitos colaterais adversos, como náusea, agitação, dores de cabeça, sonolência e disfunção sexual. A situação aponta uma necessidade maior de atenção para esse assunto vendo que pode afetar uma vida inteira. (Wallace, 2017)

Em resposta oferecemos dois tipos de tratamentos, os quais não são invasivos. Sendo um deles a acupuntura que consiste em umatécnica chinesa já comprovada com os seus efeitos analgésicos e anti- inflamatórios, isso faz com que o paciente se sinta mais disposto ao decorrer do dia.

O outro tratamento é a massagem, que conta com várias técnicas e manobras, trazendo principalmente relaxamento do paciente, fazendo com que amenise as dores e diminua o estresse do dia-a-dia.

Através de pesquisas podemos notar que o profissional de fisioterapia pode trabalhar juntamente com o profissional psicológico/psiquiatra no tratamento dessas patologias. Onde o fisioterapeuta pode atuar tanto na prevenção, como tratamento para que esses distúrbios não evoluam seus estágios.

\section{Referencia}

Abrel, F. M. Souza, F. T. \& Fagundes, S. D. Os Efeitos Da Massoterapia Sobre O Estresse Físico E Psicológico. https://revista.faema.edu.br/index.php/Revista-FAEMA/article/view/119

Almeida, S. T. (2013) Estímulos Manual E Elétrico Da Acupuntura Sistêmica No Tratamento Da Dor Crônica: Uma Revisão Sistemática. http://www.repositorio.bahiana.edu.br/jspui/bitstream/bahiana/75/1/Renato\%20Santos\%20de\%20Almeida.pdf

Barnhill, W. J. (2018) AGORAFOBIA https://pt.scribd.com/document/496005569/Transtorno-de-Ansiedade-Generalizado-TAG-Transtornos-PsiquiatricosManuais-MSD-Edicao-Para-Profissionais

Carvalho, R. J. \& Almeida, M. A. P. T. (2018), Efeitos da massoterapia sobre o sistema imunológico. Rev. Multidisc. e de Psicol. 12(40), 35366.". https://idonline.emnuvens.com.br/id/article/download/1118/1634.

Castillo, GL. R. A. Recondo, R. Asbahr, R. F. \& Manfro, G. G. (2000) Transtornos de ansiedade. https://www.scielo.br/j/rbp/a/dz9nS7gtB9pZFY6rkh48CLt/?lang=pt\#

Cordeiro, M. A. Oliveira, M. G. Renteria, M. J. \& Guimarães, A. C. (2007) Revisão Sistemática: Uma Revisão Narrativa. https://www.scielo.br/j/rcbc/a/CC6NRNtP3dKLgLPwcgmV6Gf/?format=pdf\&lang=pt

Costa, O. C. Branco, C. J. Vieira, S. I. Souza, M. D. L. \& Silva, A. R. (2019) Prevalência de ansiedade e fatores associados em adultos. https://www.scielo.br/j/jbpsiq/a/PSrDy4ZFSGDCzNgJfJwVRxz/?lang=pt\&format=html

Goyatá, S. L. T. Avelino, V. C. C. Santos, M. V. S. Junior, S. I. D. Gurgel, L. D. S. M. \& Terra, S. F. (2016) Efeitos da acupuntura no tratamento da ansiedade: $\quad$ revisão $\quad$ integrativa. $\quad$ Rev. Bras. Enferm. 69(3), https://doi.org/10.1590/00347167.2016690325i. https://www.scielo.br/j/reben/a/nFTpYKy5K4GY9JRXZ7FvcRc/?format=html\&lang=pt." 
Research, Society and Development, v. 10, n. 15, e442101523228, 2021

(CC BY 4.0) | ISSN 2525-3409 | DOI: http://dx.doi.org/10.33448/rsd-v10i15.23228

Iacoponi, E. (2000) Classificação De Transtornos Mentais E De Comportamento Da CID-10 - Diretrizes Diagnósticas E De Tratamento Para Transtornos Mentais Em Cuidados Primários. https://www.scielo.br/j/rbp/a/4ksbFDTVKW77jBjx8Cvvzkr/?lang=pt

Instituto de Psicologia - Biblioteca Dante Moreira Leite -Av. Prof. De Mello Moraes, 1721 Bloco C (2015) Tipos De Revisão De Literatura. https://www.fca.unesp.br/Home/Biblioteca/tipos-de-evisao-de-literatura

Lima, S. C. T. \& Mioto, T. C. R. (2007) Procedimentos metodológicos na construção do conhecimento científico: a pesquisa bibliográfica. https://www.scielo.br/j/rk/a/HSF5Ns7dkTNjQVpRyvhc8RR/abstract/?lang=pt\#

Minayo, S. M. Miranda, I. \& Telhado, S. R. (2021) Revisão sistemática sobre os efeitos dos probióticos na depressão e ansiedade: terapêutica alternativa. https://www.scielo.br/j/csc/a/dKmhqRf3P5d9gGfHgVnPjYR/?lang=pt

Picolli, A. (2008) O TRATAMENTO DA ANSIEDADE POR INTERMÉDIO DA ACUPUNTURA: UM ESTUDO DE CASO. https://www.scielo.br/j/pcp/a/wMxcnZXLgspCFh4qGTVcmGB/?lang=pt

Razzouk, D. (2016), Por Que O Brasil Deveria Priorizar O Tratamento Da Depressão Na Alocação Dos Recursos Da Saúde? https://www.scielo.br/j/ress/a/hffSJgnzfnWS9DHKNmv37sD/?lang=pt

Ribeiro, D. C. \& Soares, C. F. S. (2014) Desafios Para A Inserção Do Fisioterapeuta Na Atenção Básica: O Olhar Dos Gestores. https://www.scielosp.org/article/rsap/2015.v17n3/379-393/pt/

Santos, F. M. (2010) O Efeito Analgésico Da Acupuntura Na Dor Crônica E Sua Aplicabilidade No Sistema Único De Saúde: Revisão Da Literatura. https://repositorio.ufu.br/bitstream/123456789/23796/4/EfeitoAnalgesicoAcupuntura.pdf

Sartori, T. K. (2010) Atuação da naturologia para uma vida longa com qualidade". Trabalho de Conclusão de Curso na UNISUL. http://www.luzimarteixeira.com.br/wp-content/uploads/2010/12/masso-e-qualidade-de-vida.pdf

Seubert, F. \& Veronese, L. (2008) A Massagem Terapêutica Auxiliando Na Prevenção E Tratamento Das Doenças Físicas E Psicológicas. https://www.luzimarteixeira.com.br/wp-content/uploads/2010/12/masso-preventiva-pisiquica.pdf

Stopa, R. S. Malta, C. D. Oliveira, M. M. Lopes, S. C. Menezes, R. P. \& Kinoshita, T. R. (2015) Prevalência do autorrelato de depressão no Brasil: resultados da Pesquisa Nacional de Saúde, 2013. https://www.scielosp.org/article/rbepid/2015.v18suppl2/170-180/pt/

Takeda, T. O. \& Stefanelli, C. M. (2006) Atividade Física, Saúde Mental E Reabilitação Psicossocial. http://www.reme.org.br/artigo/detalhes/403

Trindade, C. P. N. (2017) Acupuntura No Tratamento Dos Transtornos Da Ansiedade. https://portalbiocursos.com.br/ohs/data/docs/227/137-

Wallace, J. K. C. \& Milev, R. (2017) Os efeitos dos probióticos nos sintomas depressivos em humanos: uma revisão sistemática. https://www.ncbi.nlm.nih.gov/pmc/articles/PMC5319175/

Wegner, F. Costa, D. A. Ribeiro, S. K. G. Andolfato, M. K. \& Comparin, A. K. (2013) Moxabustão: Uma Revisão Da Literatura. file:///C:/Users/hp/Downloads/2734-5887-1-SM.pdf 